\title{
An Improved Apparatus for Measuring Complex Viscosity of Dilute Polymer Solutions at Frequencies from 2 to $500 \mathrm{kHz}$
}

\author{
Haruhiko Nakajima, Hideho Oкамото, and Yasaku Wada \\ Department of Applied Physics, Faculty of Engineering, \\ The University of Tokyo, Bunkyo-ku, Tokyo, Japan.
}

(Received April 23, 1973)

\begin{abstract}
An apparatus which has been presented in a previous paper [Polymer J., 1, 727 (1970)] for measuring complex viscosity of dilute polymer solutions is significantly improved in both electronic circuit and vibrational assembly. As a result, the sensitivity, stability, and ease of operation are greatly increased. The frequency range is extended from 2 to $500 \mathrm{kHz}$. The method requires $10 \mathrm{cc}$ of the solution to be measured and has the advantage of being applicable even to electrically conductive solutions. Illustrative data of the complex viscosity for an aqueous solution of poly(acrylic acid) at a concentration of $0.16 \mathrm{~g} / 100 \mathrm{~m} l$ are presented.
\end{abstract}

KEY WORDS Complex Viscosity / Dynamic Viscosity / Polymer Solution / Technique / Polyelectrolyte / Poly(acrylic acid) /

As a result of numerous investigations, the complex shear viscosity of dilute polymer solutions over a wide frequency range provides us with knowledge of the dynamic behavior of polymer molecules. ${ }^{1,2}$ Several different techniques for measuring the complex viscosity of dilute polymer solutions have been presented, as reviewed by Ferry, ${ }^{1}$ Osaki, ${ }^{2}$ and McSkimin. ${ }^{3}$

A new apparatus for measuring the complex shear viscosity of liquids at frequencies from 2 to $300 \mathrm{kHz}$ has been presented in a previous paper ${ }^{4}$ by Nakajima and Wada. The techniques were based on two different principles, depending on the frequency range. In the range from 2 to $20 \mathrm{kHz}$, the frequency and decay-rate of the torsional oscillation of a long circular rod at its resonance were measured with and without a liquid applied around the rod, and the real and imaginary parts of the complex shear viscosity, $\eta^{*}=\eta^{\prime}-i \eta^{\prime \prime}$, were evaluated from the lowering of the resonant frequency and the increase of the decay-rate due to the liquid. At frequencies from 30 to $300 \mathrm{kHz}$, on the other hand, pulsed torsional waves were propagated along the same rod, and $\eta^{\prime}$ and $\eta^{\prime \prime}$ were obtained from the decrease in velocity and the increase in attenuation due to the liquid. The apparatus proved to have a high sensitıvity and was useful for measuring the complex viscosity of dilute polymer solutions over a wide frequency range. The apparatus had the advantage of being applicable even to electrically conductive solutions, such as aqueous solutions of biopolymers.

In the present paper, we wish to describe improvements of the apparatus, in the electronic circuit as well as in the mechanical section. As a result of the improvements, the frequency range was extended up to $500 \mathrm{kHz}$; at the same time, sensitivity and stability higher than the previous apparatus and increased ease of operation were attained.

\section{PRINCIPLES}

Before describing the details of the improved apparatus, the principles of the techniques employed will be briefly reviewed.

When a circular rod is excited into a torsional vibration and shear waves are propagated from the rod surface into the surrounding liquid, the force acting on a unit area of the rod surface is proportional to the velocity of the surface. The proportionality constant is defined to be the characteristic surface impedance $Z^{*}$. When the radius of the rod is much larger than the wave-length of the shear waves and the waves 
die off before they reach the wall of the vessel, $Z^{*}=R+i X$ is related to the complex shear viscosity $\eta^{*}=\eta^{\prime}-i \eta^{\prime \prime}$ of the liquid by the equations, ${ }^{5}$

$$
\begin{aligned}
\eta^{\prime} & =\frac{2 R X}{\rho \omega} \\
\eta^{\prime \prime} & =\frac{R^{2}-X^{2}}{\rho \omega}
\end{aligned}
$$

where $\rho$ is the density of the liquid and $\omega$ is the angular frequency of the vibration.

For measuring $Z^{*}$ at frequencies from 2 to $500 \mathrm{kHz}$, we employ two different techniques in an manner analogous to the previous paper: ${ }^{4}$ the torsional free-decay method up to $20 \mathrm{kHz}$ and the torsional traveling wave method at higher frequencies. First, we describe the former method.

The method developed by Mason ${ }^{5}$ utilized a quartz torsional transducer as a vibrating rod, and the resonant frequency of the transducer and the equivalent resistance are measured by the impedance method. To lower the frequency, a long resonator is necessary and, therefore, we employed a long circular rod of fused quartz with a quartz torsional transducer mounted on the top end of the rod. According to the theory of the piezoelectric transducer, ${ }^{6}$ the conversion factor $\alpha$, defined by the ratio of induced torque to applied voltage, is proportional to $\left[1-\cos \left(2 \pi l_{\mathrm{e}} / \lambda\right)\right]$, where $\lambda$ is the wavelength in the rod and $l_{\mathrm{e}}$ is the length of the electrodes along the rod axis. Since the motional electrical impedance of the resonator is proportional to $\alpha^{-2}$, the impedance becomes very large and the electromechanical coupling becomes very weak when $l_{\mathrm{e}}$ is much smaller than $\lambda$, as is the present case. When the total length of the rod (including the quartz transducer) $l=85 \mathrm{~cm}(\lambda=2 l=170 \mathrm{~cm}$ at the fundamental mode $)$ and $l_{\mathrm{e}}=5 \mathrm{~cm}$, we obtain $\alpha^{-2}=3.4 \times 10^{3}$. This would mean that the impedance method is very difficult to apply in the present case; because such a high motional impedance is inseparable from the much lower parallel impedance of the electrostatic capacity of the electrodes.

We employed, therefore, the free-decay method as described in the previous paper. ${ }^{4}$ In the previous paper we used the term "torsional reverberation method." Since this term seems somewhat inadequate, we use in this paper a new term "torsional free-decay method," abbreviated as the TFD method in the following. In this method, the lowering of the resonant frequency $\Delta f$ and the increase. of decay rate $\Delta D$ due to an applied liquid are related to $Z^{*}$ as ${ }^{4}$

$$
\begin{aligned}
& \Delta f=\frac{K}{2 \pi} X \\
& \Delta D=K R
\end{aligned}
$$

Here $K$ is an instrument constant,

$$
K=\frac{2}{\rho_{0} a}\left[\frac{h}{l}+\frac{1}{2 n \pi} \sin \left(\frac{2 n \pi h}{l}\right)\right]
$$

where $\rho_{0}, a$, and $l$ are the density, radius, and length of the rod, respectively, $h$ is the height of the liquid level from the bottom end of the rod, and $n$ is the order of the resonance.

For the high-frequency range, the torsional traveling wave method (abbreviated as the TTW method), first developed by McSkimin, ${ }^{7}$ was employed, in which the propagation constant $\gamma=$ $\alpha+i \beta$ of a torsional traveling wave along the circular rod is measured. The increases in $\alpha$ and $\beta, \Delta \alpha$ and $\Delta \beta$, arising from the applied liquid are related to $Z^{*}$ as ${ }^{7}$

$$
\begin{gathered}
\Delta \alpha=K^{\prime} R, \quad \Delta \beta=K^{\prime} X \\
K^{\prime}=\frac{2}{\rho_{0} a V}
\end{gathered}
$$

where $V$ is the velocity of torsional waves along the rod.

In the TTW method, where the wavelength $\lambda$ is very short, it is possible to adjust the electrode length $l_{\mathrm{e}}$ so as to satisfy the condition $\cos 2 \pi l_{\mathrm{e}} / \lambda=$ -1 in order to obtain the maximum value of $\alpha$ and thus the maximum electromechanical coupling.

In the TFD method, the resonance frequency $f$ of the rod is determined first so as to give the minimum decay-rate, as described in a previous paper. ${ }^{4}$ In the improved technique, a more accurate adjustment is made using the criterion that, at resonance, the decaying signal should have the same frequency as the driving signal. For this purpose, the phase of the decaying signal at a definite time $t$ (measured from the start of excitation) is compared with a continuous wave which is perfectly coherent with the driving signal. 
The decay-rate $D$ is in principle obtainable from the decay in a definite time interval. In this technique, however, it is sufficient to observe the level at $t$ alone, because the level of driving signal is kept completely constant through a series of measurements. For the same reason, it is sufficient for determination of $\alpha$ in the TTW method to measure the level of a single echo of a definite order.

The null method is employed in all the measurements by the TFD and TTW methods. The signal (the decaying signal at time $t$ in the TFD method and the $n$-th echo in the TTW method) is subtracted from a reference signal which has the frequency of the driving signal. The level and the phase of the latter are adjusted so as to give a null output. In the TTW method, the null pattern is obtained, instead of using a phase-shifter, by finely adjusting the frequency. ${ }^{8}$ The frequency difference for obtaining the null pattern with and without liquid, $\Delta f$, is related to $\Delta \beta$ as

$$
\Delta \beta=\frac{2 \pi l}{V h} \Delta f
$$

Equation 8 is straightforwardly deduced from eq 5 in a previous paper. ${ }^{4}$

In actual measurements, liquids of known $R$ and $X$ are used as references with the same level height $h$ as the test liquid. This eliminates the necessity of calculating the instrument constants, $K$ and $K^{\prime}$ in eq 5 and 7 , respectively, as well as the factor $(2 \pi l / V h)$ in eq 8 .

\section{MECHANICAL SECTION}

The vibrational assembly is basically similar to that described in a previous paper. ${ }^{4}$ The vibrator consists of a circular rod of fused quartz, $2.5 \mathrm{~mm}$ in radius and $800 \mathrm{~mm}$ in length, and a torsional quartz transducer with the same radius and $50 \mathrm{~mm}$ long which is mounted on the top of the rod. The rod and the transducer are cemented with indium solder with the axes accurately aligned, as described in a previous paper. ${ }^{4}$

The vibrational assembly has been improved in three points. As illustrated in Figure 1, the 4 electrodes for applying voltage to the transducer are not plated on the transducer but are arranged
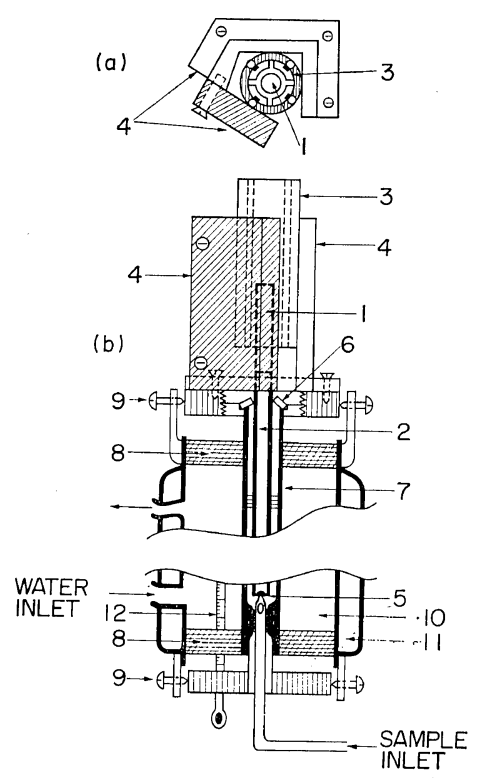

Figure 1. Mechanical part: (a) top view and (b) side view. (1) Quartz transducer, (2) vibrator rod, (3) electrodes, (4) holder of electrodes, (5) pin, (6) O-ring, (7) glass cylinder, (8) rubber blocks, (9) screws, (10) water jacket, (11) evacuated jacket, and (12) thermometer.

coaxially around the transducer with a $0.5 \mathrm{~mm}$ air gap. This enables the electrodes to be shifted vertically. The effective length of electrodes, $l_{\mathrm{e}}$, can thus be adjusted to obtain the maximum conversion factor at the highest frequency, 530 $\mathrm{kHz}$, where, if not adjusted, the conversion factor might be too low. For obtaining a purely torsional wave in the TTW mode, it is important to set the central lines of the electrodes exactly at $\pm 45^{\circ}$ with respect to the $y$-axis of the quartz.

The rod is supported with a metal pin at the bottom and with an O-ring of silicone rubber higher up. The coaxial glass cylinder has a gap of $c a .0 .5 \mathrm{~mm}$ from the rod surface and liquid fills this gap up to an appropriate level. The cylinder is supported by two rubber blocks. To obtain a uniform gap separation along the rod, the cylinder is slightly bent by adjusting screws at the top and the bottom.

Since mechanical characteristics of the rod vary with temperature, the temperature of the rod is required to be constant within $\pm 0.005 \mathrm{~K}$. The cylinder containing the liquid is surrounded 
with a water-jacket, which is further covered with an evacuated jacket. Water at a constant temperature is circulated through the water jacket.

\section{ELECTRONIC CIRCUIT}

Figure 2 illustrates a block diagram of the overall circuitry of the apparatus.

A frequency-synthesizer (1) generates highfrequency waves at a frequency $f^{*}$, which is $c a$. $1.5 \mathrm{MHz}$. The frequency is variable digitally and can be continuously varied within a range of $100 \mathrm{~Hz}$. In the carrier generator (2), the frequency $f^{*}$ of the synthesizer output is divided into carrier frequencies $f$ and $2 f$ with the flip-flops: $f=f^{*} / N$, where $N$ is an even integer. Since $f^{*}$ is controlled within $1 \mathrm{~Hz}, f$ is adjustable within $1 / \mathrm{N} \mathrm{Hz}$.

The carrier frequency is variable by changing $N$. In this apparatus, seven freqnencies are used: (a) $2.2 \mathrm{kHz}$, (b) $6.6 \mathrm{kHz}$, and (c) $19.8 \mathrm{kHz}$ in the TFD mode, and (d) $45.3 \mathrm{kHz}$, (e) $125 \mathrm{kHz}$,

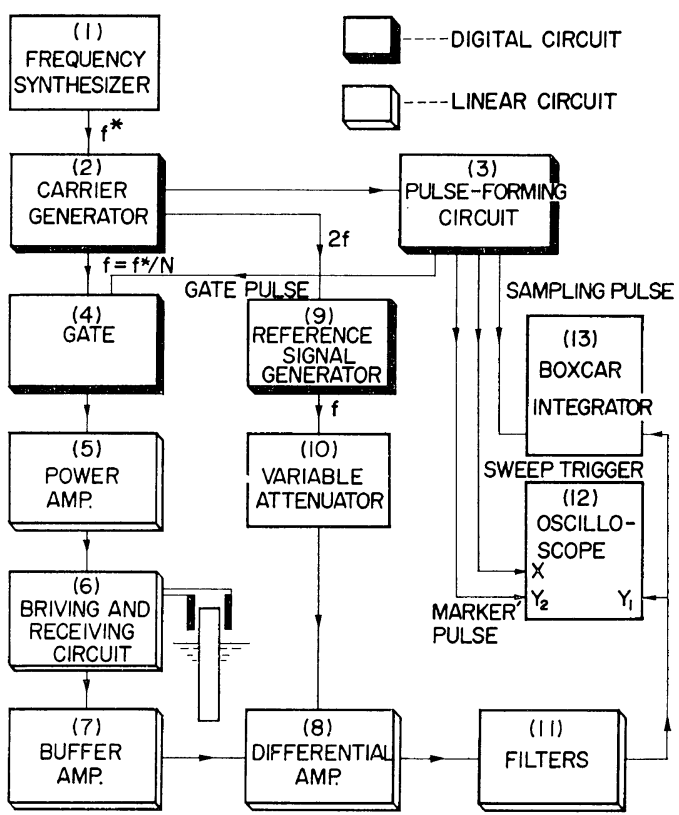

Figure 2. Block diagram of electronic circuit. (f) $350 \mathrm{kHz}$, and (g) $530 \mathrm{kHz}$ in the TTW mode.

\section{SECTION B}

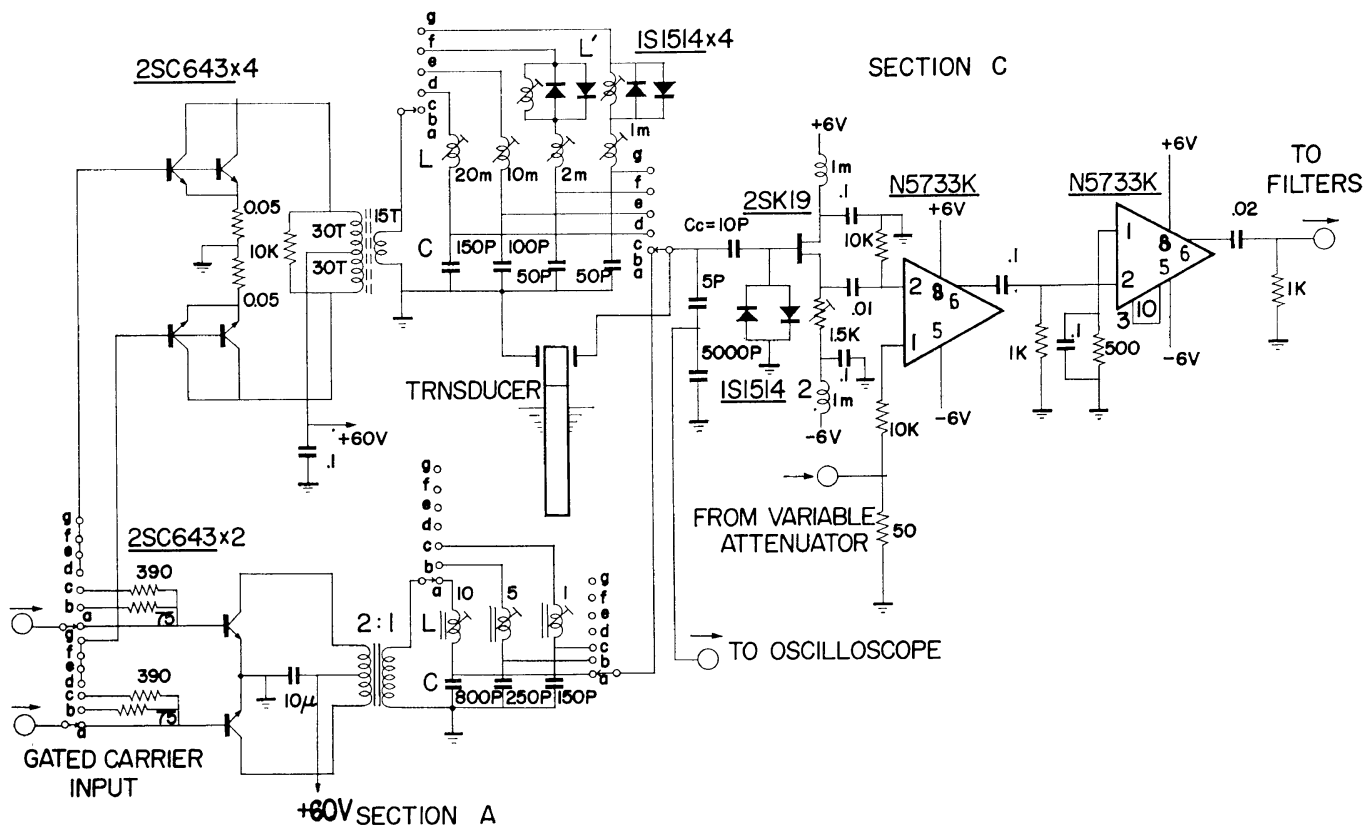

Figure 3. Circuit of power amplifiers, driving and receiving circuits (section $\mathrm{A}$ for channels a to $\mathrm{c}$ and section B for channels $\mathrm{d}$ to $\mathrm{g}$ ), buffer amplifier, and differential amplifier (section C). Resistance in $\Omega$, capacitance in $\mathrm{F}$, and inductance in $\mathrm{H}$. 
In the TFD mode, the three frequencies correspond to resonances of the rod of order $n=1$, 3 , and 9, respectively. All the timing pulses of this apparatus are formed in a pulse-forming circuit (3) in synchronization with the carrier.

The carrier in the form of a square wave is gated in (4) by gate pulses with a width of $T_{\mathrm{d}}$ and a repetition period $T_{\mathrm{p}}$, both of which are adjustable. In the TFD mode, $T_{\mathrm{d}}$ must be long enough so that the vibration of the rod may reach a stationary state and $T_{\mathrm{p}}$ must be sufficiently long so that the decay may reach the noise level by the start of the subsequent excitation. Since the time constant for the build-up of mechanical vibration is similar to that of free decay, $T_{\mathrm{d}}$ is chosen as $T_{\mathrm{p}} / 2$. In the TTW mode $T_{\mathrm{p}}$ is adjusted so that the echo train may decay to the noise level by the subsequent driving pulse.

The gate circuit (4) consists of multistage nand gates and diminishes the leakage signal to the noise level.

A power amplifier (5) is followed by a driving circuit (6), consisting of an $L-C$ resonant circuit for each channel, which feeds pulsed sinusoidal waves of frequency $f$ to the quartz transducer. The peak-to-peak voltage across the transducer electrodes reaches 4,000 volts at the maximum.

The signal of free decay (TFD mode) or multiple echoes (TTW mode) is received by a receiving circuit (6) and fed through a buffer amplifier (7) to a differential amplifier (8). Detailed circuits of (5) to (8) are drawn in Figure 3, where section A consists of power amplifier and driving and receiving circuit for channels (a) to (c) (TFD mode), and section B those for channels (d) to (g) (TTW mode).

In section $\mathrm{A}$, the output (square wave) of a push-pull power amplifier $(2 \mathrm{SC} 643 \times 2)$ is fed through an output transformer (iron core, $2: 1$ ) to series $L C$ circuits. The sinusoidal voltage across $C$ is applied to the quartz transducer. This voltage is constant because the input level to the amplifier is kept constant and at the same time the power amplifier works at its saturated level. The voltage is measured, when necessary, with an oscilloscope.

In section $\mathrm{B}$, the output (square wave) of a parallel push-pull power amplifier $(2 \mathrm{SC} 643 \times 4)$ is fed through an output transformer (ferrite core, $4: 1)$ to series $L C$ circuits.

During the driving period, high-speed diodes $(1 \mathrm{~S} 1514 \times 2)$ in the input circuit of the buffer amplifier (2SK19) become highly conductive and protect the amplifier. As a consequence, the coupling capacitor $C_{\mathrm{c}}$ is added to $C$ only at the driving period and shifts the resonant frequency. of the $L C$ circuits. This effect is not negligible at the highest frequency channels (f) and (g), and, to compensate the effect, small inductances

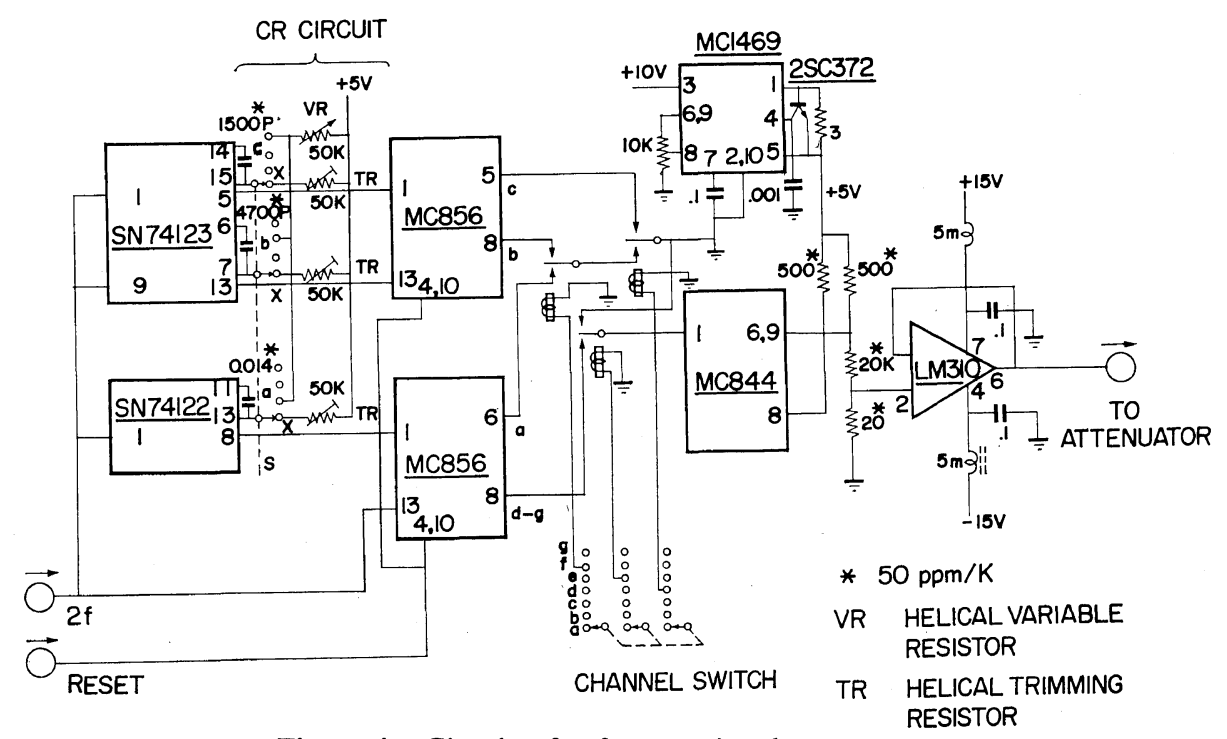

Figure 4. Circuit of reference signal generator. 
$T_{p}=m / f$ (A) 'CARRIER WAVE

(B) GATE PULSE

(c) GATED CARRIER

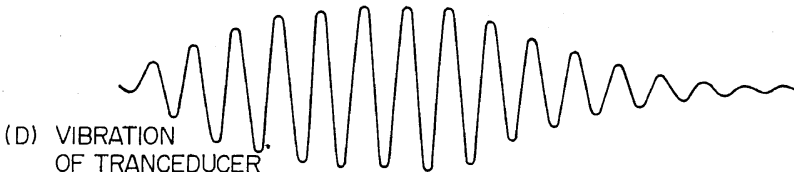

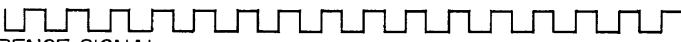
(E) REFERENCE SIGNAL

(F) FILTER OUTPUT

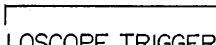

(G) OSCILLOSCOPE TRIGGER

(H) OSCILLOSCOPE MARKER (1) SAMPLING SIGNAL TO BOXCAR
INTEGRATOR

(a)
$T_{p}=m / f$

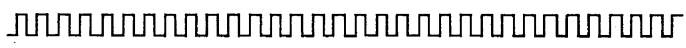
$\stackrel{T_{d}}{\longrightarrow}$

л됴

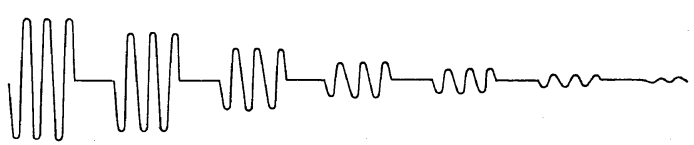

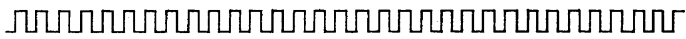

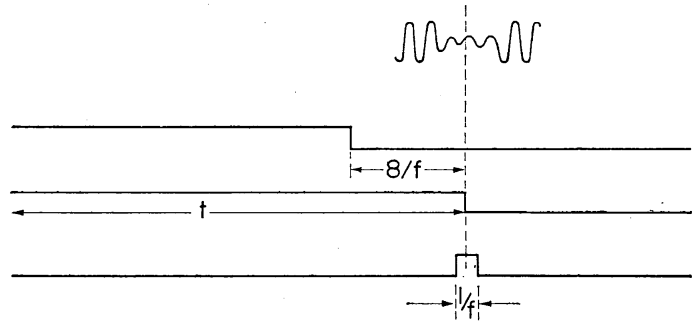

(b)

Figure 5. Schematic illustration of signals in (left) torsional free-decay mode and (right) torsional traveling wave mode. 
$L^{\prime}$, which are effective only during the receiving period, are added.

Section $C$ in Figure 3 includes the buffer amplifier (7) (FET source follower, 2SK19) and the differential amplifier (8) (N5733K), followed by a video-amplifier (N5733K). The reference signal to the differential amplifier, a continuous square wave having the carrier frequency $f$, is generated in a reference signal generator (9) and is attenuated to an appropriate level with an variable attenuator (10) of $0.1 \mathrm{db}$ step.

The circuit of the reference signal generator (9) is given in Figure 4. The function of the reference signal generator is to generate a continuous square wave at a frequency $f$ with an adjustable phase and a definite level. For this purpose, the input square wave signal from (2) with a frequency $2 f$ triggers a TTL monostable multivibrator (SN74122, SN74123) and produces $2 f$ pulses with a variable duty time. The duty time is varied by adjusting the time constant of the $C R$ circuits with helical trimming resistors. The $2 f$ pulses thus obtained trigger the flip-flops (MC856 $\times 2$ ), which generate $f$ pulses of $50-\%$ duty time. The level of this reference signal is made constant with a power gate (MC844), the output of which possesses of constant logical 1 level of $c a .5 \mathrm{~V}$ (the temperature coefficient of the level is diminished with a voltage regulator, MC1469-R) and a low value $(0.1 \mathrm{~V}, 0.2 \mathrm{mV} / \mathrm{K})$ of logical 0 level. As a result, the amplitude of the reference signal proved to be constant within $\pm 0.04 \%$, even when the temperature varied by $10^{\circ} \mathrm{K}$. A voltage follower (LM310) functions as a constant voltage source to the attenuator. This eliminates the effect of temperature variation on the attenuator.

The output from (8), the difference signal of the two inputs from (7) and (10), is fed to bandpass filters (11), which consist of three active filters for channels (a) to (c) and four $L C$ filters for channels (d) to $(\mathrm{g})$. The temperature of these filters is kept constant within $\pm 1^{\circ} \mathrm{K}$ to avoid any drift of the band-pass frequency.

The output signal of the filters (11) is displayed on the oscilloscope (12). To eliminate the error arising from noise and uncertainty in determining the zero level, a boxcar integrator (13) is employed which indicates the average level within 1 to $10 \mathrm{sec}$. The oscilloscope serves as an in- dicator of the null pattern for a coarse adjustment and the boxcar integrator is useful for fine adjustment.

All the timing signals for operation of the system are formed in (3), as schematically illustrated in Figure 5. The trigger to the oscilloscope sweep is formed at $8 / f$ before $t$ at which the signal is measured. As a result, 16 waves are displayed on the screen and a marker at time $t$ appears at the center. The sampling signal to the boxcar integrator continues from $t-(1 / 2 f)$ to $t+(1 / 2 f)$.

\section{PERFORMANCE OF TFD MODE}

In the TFD mode, the pulse width $T_{\mathrm{d}}$ of the driving signal is chosen as $50 \%$ of the repetition period $T_{\mathrm{p}}$. The criterion for setting the value of $T_{\mathrm{p}}$ has been described in the preceding section; $T_{p}$ in the case of dilute aqueous solutions was ca. $10^{2} \mathrm{msec}$ in order of magnitude. The time $t$ at which the decay signal is measured was determined in such a manner that the signal would have a level about $40 \mathrm{db}$ higher than the noise level.

After setting the values of $T_{\mathrm{d}}$ and $T_{\mathrm{p}}$, the phase shift in the reference signal generator is adjusted so that the ouptut will have just the same phase as the decaying signal at resonance. For this purpose, three trimming resistors in the $C R$ circuit of the reference signal generator are adjusted in the following manner.

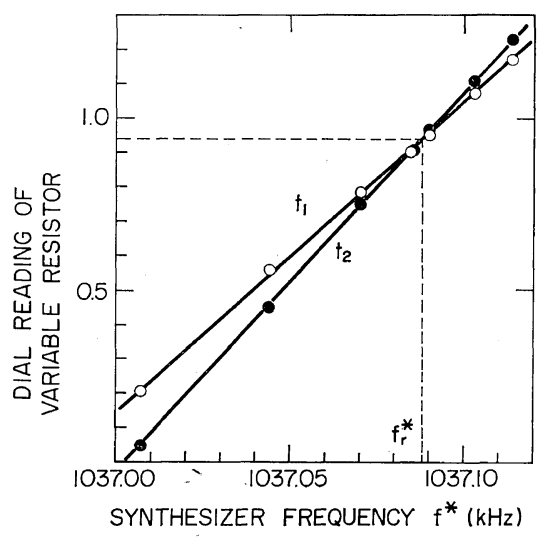

Figure 6. Example of phase-shift adjustment of the reference signal generator for channel (c). The level difference is $3.35 \mathrm{db}$ between $t_{1}$ and $t_{2}\left(t_{1}<t_{2}\right)$. 
To give an appropriate damping, a liquid, water for example, is applied to the rod. Two times, $t_{1}$ and $t_{2}$, are arbitrarily selected. The decay signals at $t_{1}$ and $t_{2}$ for each carrier frequency are balanced against the reference signal by adjusting its level and phase with the attenuator and the phase shifter. An example of such a measurement is illustrated in Figure 6 in which the dial reading of the variable resistor is plotted against the frequency of the synthesizer output $f^{*}$. Two straight lines cross at a frequency $f_{\mathrm{r}}{ }^{*}$ which gives the resonant frequency $f_{\mathrm{r}}=f_{\mathrm{r}}{ }^{*} / N$. After finding $f_{\mathrm{r}}$, the switch $\mathbf{S}$ (Figure 4) is turned to $X$ and the trimming resistor is set at the same resistance as the variable resistor at this cross point. This setting was found to be stable through a series of measurements which lasted over several months.

Calibration curves of $\Delta f$ and $\Delta D$ against $R$ and $X$ are obtained with reference liquids of known viscosity. Low-molecular-weight liquids are usually suitable as references because they generally possess no relaxaxations in the frequency range of interest, and $R$ and $X$ are related to the steady-flow viscosity $\eta$ and the density $\rho$ as

$$
R=X=\sqrt{\frac{\omega \rho \eta}{2}}
$$

In our case, where aqueous solutions of polymers are concerned, aqueous solutions of sucrose with different concentrations (containing a trace of salicylic acid as a preservative) were utilized as references. Examples of calibration curves for channel (b) are illustrated in Figure 7, where the ordinates express the values of $f^{*}$ and the attenuator reading when the signal at a definite time $t$ is balanced against the reference signal.

In actual measurements for polymer solutions, calibration curves, illustrated in Figure 7 as examples, are used and $R$ and $X$ of polymer solutions are obtained directly from $f^{*}$ and $N$. The slope of the calibration curves, which are very nearly straight lines, is quite reproducible, but the curves in some cases shift vertically during measurements, probably because of contamination of the rod surface. Therefore, checks of the calibration curves at one point are necessary within a series of measurements. Theoretically, the calibration of $f^{*} v s . \sqrt{\rho \eta}$ should be

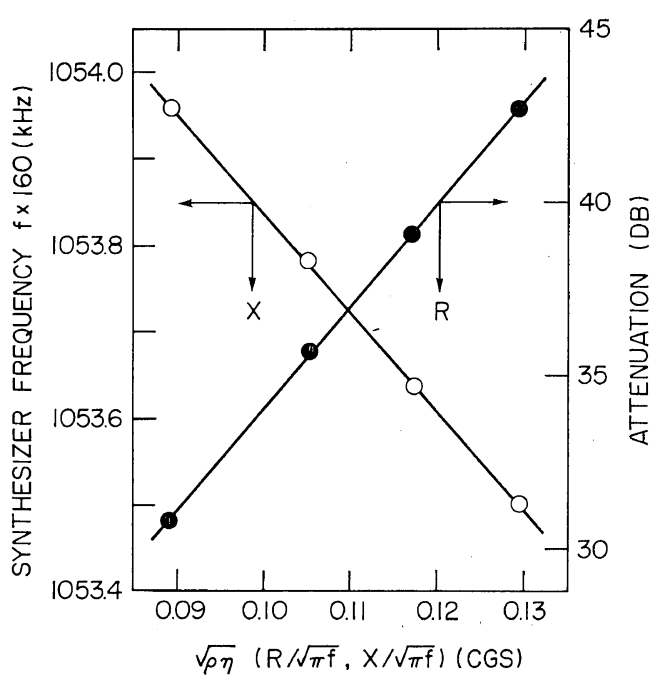

Figure 7. Example of calibration curves of channel (b) $(6.59 \mathrm{kHz})$ obtained for sucrose solution, $h=$ $70 \mathrm{~cm}, 30^{\circ} \mathrm{C}$.

linear, while that of attenuation $v s . \sqrt{\rho \eta}$ should be slightly curved because, since the driving voltage is kept constant, the amplitude at the start of decay is not constant but depends on $\sqrt{\rho \eta}$. The curve in Figure 7 indicates, however, that this nonlinearity is very small.

The liquid-level height $h$ is to be selected at an appropriate value because, as has been described in a previous paper, ${ }^{4}$ bending oscillation of the rod would be excited if $h$ is poorly chosen. The suitable value of $h$ common for three channels of the TFD mode was $70 \mathrm{~cm}$ in our case. Wetting of the rod surface above the liquid level has an appreciable effect on the data and is to be avoided.

\section{PERFORMANCE OF TTW MODE}

The criterion of selection of pulse width and repetition period has been described in the preceding section. In the case of dilute aqueous solutions, $T_{\mathrm{d}}$ was about $0.3 \mathrm{msec}$ and $T_{\mathrm{p}}$ about $300 \mathrm{msec}$.

The order $n$ of the echo to be observed is determined such that the echo has a level of $c a .40 \mathrm{db}$ over the noise. The phase and level of the echo are measured by balancing it against the reference signal in a manner analogous to 


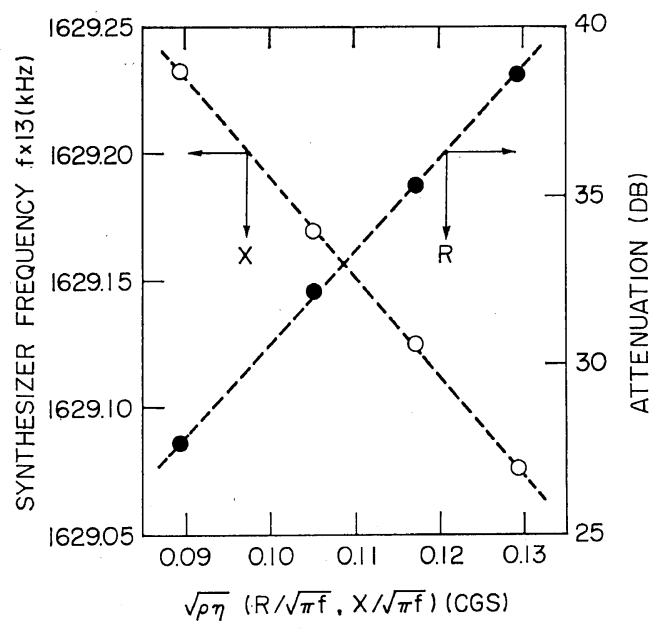

Figure 8. Example of calibration curves of channel (e) $(125.3 \mathrm{kHz})$ obtained for sucrose solutions, $h=$ $70 \mathrm{~cm}, 30^{\circ} \mathrm{C}$.

the TFD mode. The balancing of the echo becomes sharper with increasing $n$.

In the TTW mode, a variation of the carrier frequency is made for balancing, without employing a phase shifter as has been described in the preceding section.

In Figure 8 is shown an example of the calibration curves for channel (e) obtained by use of the sucrose solutions as references. The ordinates indicate synthesizer frequency $f^{*}$ and

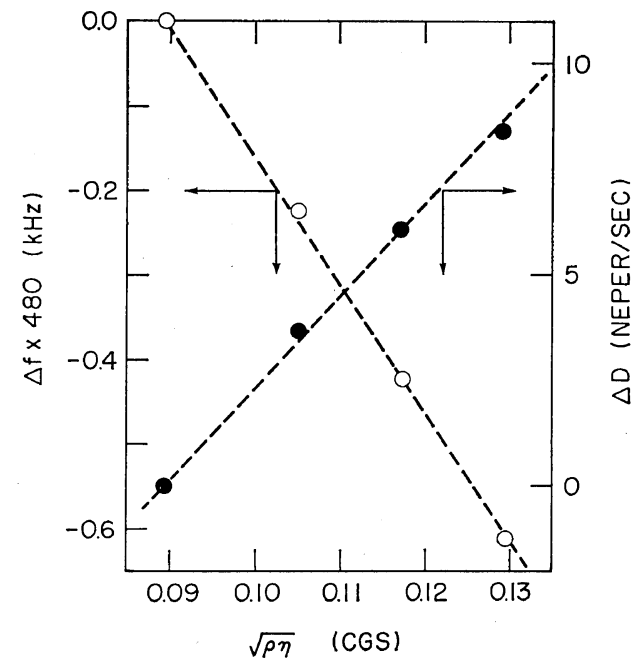

Figure 9. Decrease in resonant frequency and increase of decay-rate for sucrose solutions (excess over water) at $2.17 \mathrm{kHz}, h=70 \mathrm{~cm}, 30^{\circ} \mathrm{C}$. Broken lines represent calculated values.

attenuator reading for balancing of the 38th echo. The echo as a whole cannot be made null because the echo envelope is not of a perfectly square form. The center of the echo is therefore balanced in the measurement. The reproducibility of the calibration curves is the same as in the TFD mode.

The selection of liquid level $h$ is not critical

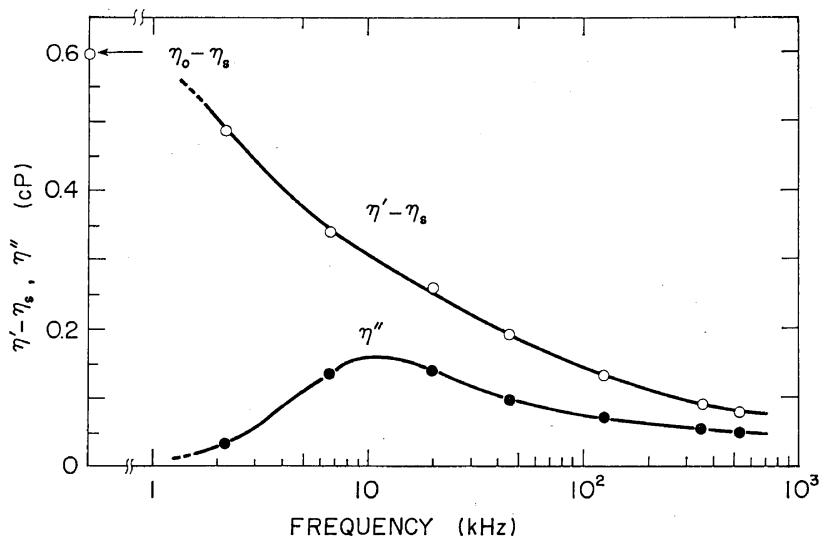

Figure 10. Real and imaginary parts of complex viscosity $\eta^{\prime}-i \eta^{\prime \prime}$ of a poly(acrylic acid) $($ D.P. $=1300)$ in water at $30^{\circ} \mathrm{C}$. Polymer concentration= $0.159 \mathrm{~g} / 100 \mathrm{ml}, \quad \mathrm{NaCl}$ concentration $=0.01 \mathrm{M}$, degree of neutralization $(\mathrm{NaOH})=0.18$. $\eta_{0}$ and $\eta_{s}$ represent steady-flow viscosities of solution and solvent, respectively. 
in the TTW mode and hence it is convenient to use the value of $h$ common to the TFD mode.

\section{ILLUSTRATIVE DATA}

The absolute accuracy of the TFD method is demonstrated in Figure 9, where the agreement between theory and experiment is shown for excess values of $\Delta f$ and $\Delta D$ in sucrose solution over water. The broken lines represent values calculated from eq 3 and 4 by using values of $h=70 \mathrm{~cm}, a=2.5 \mathrm{~mm}, l=85 \mathrm{~cm}$, and $\rho_{0}=2.2 \mathrm{~g} /$ $\mathrm{cm}^{3}$. The absolute accuracy of the TTW method has been described in a previous paper $^{4}$ and therefore is not reproduced here.

With the apparatus described in this paper, we measured $\eta^{\prime}$ and $\eta^{\prime \prime}$ of dilute aqueous solutions of poly(acrylic acid) and poly(methacrylic acid) as functions of degree of neutralization, salt concentration, and molecular weight of polymers. Detailed results will be published in other papers, but one example is illustrated in Figure 10.

\section{REFERENCES}

1. J. D. Ferry, Viscoelastic Properties of Polymers, 2nd ed., John Wiley, New York, N. Y., 1969.

2. K. Osaki, "Advances in Polymer Science" (in press).

3. H. J. McSkimin, "Physical Acoustics," Vol. 1A, W. P. Mason, Ed., Academic Press, New York, N. Y., 1964.

4. H. Nakajima and Y. Wada, Polymer J., 1, 727 (1970).

5. W. P. Mason, Trans. ASME, 69, 359 (1947).

6. W. P. Mason, "Piezoelectric Crystals and Their Application to Ultrasonics," D. Van Nostrand, New York, N. Y., 1950.

7. H. J. McSkimin, J. Acoust. Soc. Amer., 24, 355 (1952).

8. H. J. McSkimin, and P. Andreatch, Jr., ibid., 42, 248 (1967). 